\title{
On Algebraically Integrable Differential Operators on an Elliptic Curve
}

Pavel ETINGOF ${ }^{\dagger}$ and Eric RAINS $\ddagger$

$\dagger$ Department of Mathematics, Massachusetts Institute of Technology, Cambridge, MA 02139, USA

E-mail: etingof@math.mit.edu

URL: http://www-math.mit.edu/ etingof /

$\ddagger$ Department of Mathematics, California Institute of Technology, Pasadena, CA 91125, USA E-mail:rains@caltech.edu

Received April 25, 2011, in final form June 30, 2011; Published online July 07, 2011

doi:10.3842/SIGMA.2011.062

\begin{abstract}
We study differential operators on an elliptic curve of order higher than 2 which are algebraically integrable (i.e., finite gap). We discuss classification of such operators of order 3 with one pole, discovering exotic operators on special elliptic curves defined over $\mathbb{Q}$ which do not deform to generic elliptic curves. We also study algebraically integrable operators of higher order with several poles and with symmetries, and (conjecturally) relate them to crystallographic elliptic Calogero-Moser systems (which is a generalization of the results of Airault, McKean, and Moser).
\end{abstract}

Key words: finite gap differential operator; monodromy; elliptic Calogero-Moser system 2010 Mathematics Subject Classification: 35J35; 70H06

To Igor Moiseevich Krichever on his 60th birthday

\section{Introduction}

In this paper we study differential operators

$$
L=\partial^{n}+a_{2}(z) \partial^{n-2}+\cdots+a_{n}(z)
$$

which are algebraically integrable (i.e., there exists a nonzero differential operator $M$ of order relatively prime to $L$ such that $[L, M]=0$ ). Such operators were first studied in [2] and became a focus of attention since the seventies, as they provide explicit solutions to the Gel'fand-Dickey hierarchy (in particular, the KdV hierarchy for $n=2$ and the Boussinesq hierarchy for $n=3$; see $[6,7]$ and references therein). A general classification of such operators was obtained in [13].

We are interested in making this classification more explicit in the special case when the coefficients $a_{i}(z)$ are meromorphic functions on an elliptic curve $E$. For instance, in the simplest nontrivial case $n=2$ and a single pole, it is well known that the only algebraically integrable operator, up to equivalence, is the Lamé operator

$$
L=\partial^{2}-m(m+1) \wp(z),
$$

where $m$ is an integer; its algebraic integrability was discovered by Hermite. In the case $n=2$ and multiple poles, the answer is much more interesting (see [4, Section 4.1], as well as [10, 11] and references therein).

\footnotetext{
${ }^{\star}$ This paper is a contribution to the Special Issue "Relationship of Orthogonal Polynomials and Special Functions with Quantum Groups and Integrable Systems". The full collection is available at http://www.emis.de/journals/SIGMA/OPSF.html
} 
We study the problem of classification of algebraically integrable operators $L$ for $n>2$. It turns out that already in the case $n=3$ and a single pole, the situation is much richer and more complicated than for $n=2$; in particular, there exist algebraically integrable operators $L$ of third order with one pole defined on an infinite family of special elliptic curves over $\mathbb{Q}$, which do not deform to operators (with one pole) on a generic elliptic curve. We provide a list of third order algebraically integrable operators with one pole which is conjecturally complete, and state some results and conjectures concerning operators with several poles. In particular, we conjecture that in the special case of operators with symmetries, algebraically integrable operators are described in terms of the classical crystallographic elliptic Calogero-Moser systems introduced in [8].

The paper is organized as follows. In Section 2, we give an exposition of the general theory of algebraically integrable operators, in particular those on an elliptic curve; the results here are mostly well known, but for reader's convenience we give an exposition based on differential Galois theory similar to one in [4]. In this section we also propose a general conjecture on the classification of operators with one pole. In Section 3, we present computational results for third order algebraically integrable operators with one pole, and give a conjectural classification of such operators. Finally, in Section 4 we discuss operators with several poles, and state a conjecture on the connection with the systems of [8].

\section{The general theory of algebraically integrable operators}

In this subsection we review the basics on algebraically integrable ordinary differential operators. Most of this material is well known; we refer the reader to $[13,16,15,17,10,4,5,11]$ and references therein.

\subsection{Definition of algebraic integrability and meromorphicity of coefficients}

Consider the differential operator

$$
L=\partial^{n}+a_{1}(z) \partial^{n-1}+\cdots+a_{n}(z),
$$

where $a_{i}(z)$ are smooth functions on some interval in $\mathbb{R}$. Note that the coefficient $a_{1}$ can be gauged away by conjugation of $L$ by $e^{\frac{1}{n} \int a_{1}(z) d z}$. So without loss of generality, we may (and will) assume that $a_{1}=0$, i.e.

$$
L=\partial^{n}+a_{2}(z) \partial^{n-2}+\cdots+a_{n}(z) .
$$

Recall that $L$ is called algebraically integrable (or algebro-geometric) if there exists a nonzero differential operator $M$ of order relatively prime to $L$ such that $[L, M]=0$ [2]. Note that up to scaling $M$ is necessarily monic (i.e., has leading coefficient 1 ).

For example, if $n=2$ then $L=\partial^{2}+u(z)$, and if $L$ is algebraically integrable then $u$ is called a finite-gap potential.

Theorem 2.1 ([16, Theorem 6.10], see also [13]). If $L$ is algebraically integrable, then $a_{i}(z)$ extend to meromorphic functions on the complex plane. Moreover, the order of each pole of the function $a_{i}$ in $\mathbb{C}$ is at most $i$, for $i=2, \ldots, n$; in other words, the operator $L$ has regular (or Fuchsian) singularities in $\mathbb{C}$.

\section{$2.2 \quad$ The indices of $L$}

Let $a_{i}(z)=b_{i} z^{-i}(1+O(z))$ near $z=0$. Then by rescaling $z$ the operator $L$ can be degenerated into the operator with rational coefficients

$$
L_{0}=\partial^{n}+b_{2} z^{-2} \partial^{n-2}+\cdots+b_{n} z^{-n}=0 .
$$


Consider the differential equation

$$
L_{0} z^{m}=0 .
$$

This equation is equivalent to the algebraic equation

$$
P_{L}(m)=0,
$$

where

$$
P_{L}(m)=m(m-1) \cdots(m-n+1)+b_{2} m(m-1) \cdots(m-n+3)+\cdots+b_{n} .
$$

Let $m_{j}, j=0, \ldots, n-1$, be the roots (with multiplicities) of the polynomial $P_{L}$, i.e.

$$
P_{L}(s)=\left(s-m_{0}\right) \cdots\left(s-m_{n-1}\right) .
$$

The numbers $m_{j}$ are called the indices of $L$ at 0 . They are arbitrary numbers satisfying the relation

$$
\sum_{j=0}^{n-1} m_{j}=n(n-1) / 2 .
$$

Obviously, the indices uniquely determine the coefficients $b_{i}$.

Similarly, one defines the indices of $L$ at any point $z_{0}$.

Example 2.2. The indices of $L$ at a regular point are $0,1, \ldots, n-1$.

\subsection{Algebraic integrability of homogeneous rational operators}

Proposition 2.3. The operator $L_{0}$ given by formula (2.1) is algebraically integrable if and only if the indices $m_{j}$ are integers which are distinct modulo $n$.

Proof. It is easy to see (see e.g. [3]) that the operator $L_{0}$ is algebraically integrable if and only if the equation $L_{0} \psi=\mu^{n} \psi$ admits a Baker-Akhiezer solution of the form $F(\mu z)$, where

$$
F(x)=e^{x} Q(1 / x),
$$

and $Q$ is a polynomial such that $Q(0)=1 .^{1}$ Solving the equation by the power series method, we see that this happens if and only if $m_{j}$ are distinct modulo $n$ (and thus represent each residue class exactly once).

Corollary 2.4. If $L$ is algebraically integrable then the indices $m_{j}\left(z_{0}\right)$ of $L$ at every point $z_{0}$ are integers which are distinct modulo n.

Proof. Suppose $L$ is algebraically integrable. Since by Theorem 2.1, the commuting operator $M$ has regular singularities at $z_{0}$, its rational degeneration $M_{0}$ is well defined, and commutes with $L_{0}$, so $L_{0}$ is algebraically integrable. So the result follows from Proposition 2.3.

When $m_{j}$ are integers, we will order them in the increasing order, $m_{0} \leq m_{1} \leq \cdots \leq m_{n-1}$. It is also convenient to introduce the "gaps" $q_{j}:=m_{j}-m_{j-1}, j=1, \ldots, n-1$, which clearly determine $m_{j}$. In the integrable case, these are nonnegative integers not divisible by $n$.

\footnotetext{
${ }^{1}$ E.g., if $L_{0}$ is algebraically integrable, then it admits a homogeneous commuting operator $M_{0}$ of relatively prime order $m$ such that $L_{0}^{m}=M_{0}^{n}$, and the system of differential equations $L_{0} \psi=\mu^{n} \psi, M_{0} \psi=\mu^{m} \psi$ can be reduced to a first order scalar equation, which has a solution of the required form by Euler's formula.
} 


\subsection{Algebraic integrability of differential operators on elliptic curves}

Let $\Gamma \subset \mathbb{C}$ be a lattice, and $E=\mathbb{C} / \Gamma$ be the corresponding elliptic curve. Assume that $a_{i}(z)$ are rational functions on $E$ (i.e., elliptic functions).

Theorem 2.5. The following conditions on $L$ are equivalent.

(i) $L$ is algebraically integrable.

(ii) The monodromy of the equation

$$
L \psi=\lambda \psi
$$

around every pole of $L$ in $E$ is trivial for any eigenvalue $\lambda \in \mathbb{C}$.

(iii) The monodromy group of equation (2.2) is upper triangular in some basis.

(iv) For generic $\lambda$ (i.e., outside of finitely many values), equation (2.2) has a basis of solutions of the form

$$
\psi(z)=e^{\beta z} \prod_{i=1}^{m} \frac{\theta\left(z-\alpha_{i}\right)}{\theta\left(z-\beta_{i}\right)},
$$

where $\theta$ is the first Jacobi theta-function.

Proof. $(i) \Longrightarrow(i i)$. Since $L$ is algebraically integrable, by Corollary 2.4, the indices of $L$ at every pole are integers. Hence the monodromy matrices of equation (2.2) around the poles of $L$ are unipotent. Also, it follows from [3] that the differential Galois group of (2.2) for generic $\lambda$ is an algebraic torus. Since monodromy matrices belong to the differential Galois group, and since every unipotent element of a torus is trivial, we conclude that the monodromy matrices of (2.2) around the poles of $L$ are trivial for generic, hence for all $\lambda$.

(ii) $\Longrightarrow$ (iii). If (ii) holds, the monodromy group of (2.2) is Abelian, hence is upper triangular in some basis.

$($ iii $) \Longrightarrow($ iv $)$. Assume (iii) holds. Since $L$ has regular singularities, the differential Galois group of equation (2.2) is the Zariski closure of the monodromy group. Hence the differential Galois group of equation (2.2) is triangular as well. But it is shown in [3] that the differential Galois group of (2.2) is reductive for generic $\lambda$. Hence this group is Abelian, and is a torus for generic $\lambda$. Thus there is a fundamental system of solutions of the form (2.3) (see [3]).

$(i v) \Longrightarrow(i)$. If $(i v)$ holds, then the monodromy of equation (2.2) around poles is trivial for generic $\lambda$. Hence it is trivial for all $\lambda$, and the monodromy group, hence the differential Galois group of (2.2) is Abelian. So by [3], there exists a nonzero differential operator $M$ of order coprime to the order of $L$ such that $[L, M]=0$.

\section{Remark 2.6.}

1. This theorem is similar to Theorem 5.9 in [5], which goes back to [4].

2. A similar theorem, with the same proof, holds in the trigonometric (nodal) and rational (cuspidal) case, i.e., when the coefficients of $L$ are rational functions on the nodal or cuspidal curve of arithmetic genus 1 which are regular at infinity. More precisely, in the rational case, since the singularity at infinity is irregular, we must add the Stokes matrix at infinity to the monodromy group, and we should also replace $\theta(z)$ with $\sin (z)$ and $z$ in the trigonometric and rational cases, respectively. We note that in the trigonometric and rational case, the implication $(i v) \Longrightarrow(i)$ of Theorem 2.5 was proved in [19].

\subsection{Operators with one pole}

In this subsection we will consider the special case when $L$ has only one pole, at the point $0 \in E$. 


\subsubsection{Second order operators with one pole}

Let $n=2$, and let $L$ have a unique pole at 0 (the simplest nontrivial case). In this case, up to an additive constant, the operator $L$ has the form

$$
L=\partial^{2}+a \wp(z),
$$

where $\wp$ is the Weierstrass function of $E$ (the Lamé operator). Local analysis near 0 (i.e., the condition that the local monodromy is trivial) shows that algebraic integrability of such $L$ implies that $a=-m(m+1)$, where $m$ is a nonnegative integer. Conversely, it was shown by Hermite that if $a=-m(m+1)$ then $L$ is indeed algebraically integrable. Namely, the triviality of the monodromy of (2.2) near 0 is easy to see by noting that the operator $L$ is $\mathbb{Z}_{2}$-invariant, while the indices at 0 are $-m$ and $m+1$, whose difference is odd; thus, the algebraic integrability of $L$ follows from Theorem 2.5.

\subsubsection{The algebraic integrability locus}

Assume now that we have fixed the indices $m_{j}$ distinct modulo $n$ (and thus the coefficients $b_{i}$ ). Then the set of possible operators $L$ is parametrized by the Laurent coefficients $a_{i k}$ of $a_{i}(z)$ of nonpositive degrees $-k, k<i$, and also by the coefficients $g_{2}, g_{3}$ of the differential equation

$$
\left(\wp^{\prime}\right)^{2}=4 \wp^{3}-g_{2} \wp-g_{3}
$$

for the Weierstrass function $\wp$ of the elliptic curve $E$. Note that we have the $\mathbb{C}^{*}$-action rescaling the lattice $\Gamma$, with respect to which these parameters have the following degrees (or weights):

$$
\operatorname{deg}\left(g_{2}\right)=4, \quad \operatorname{deg}\left(g_{3}\right)=6, \quad \operatorname{deg}\left(a_{i k}\right)=i-k .
$$

Thus, we will think of these parameters as homogeneous coordinates on the weighted projective space with these weights, and define the algebraic integrability locus $X_{\mathbf{m}}$ (for each choice of indices $\mathbf{m}$ ) as a subset of this weighted projective space.

\subsubsection{The cyclically symmetric operators}

For every choice of the indices $\mathbf{m}$, there exists a unique operator $L$ such that all the coefficients $a_{i k}$ equal zero. Let us denote this operator by $L(0)$.

Proposition 2.7. For $n \geq 2$, the operator $L(0)$ is algebraically integrable (for any indices distinct modulo $n$ ) in the following cases:

(i) $n=2$ (the Lamé operator any elliptic curve);

(ii) $n=3,6, g_{2}=0$ (equianharmonic elliptic curve);

(iii) $n=4, g_{3}=0$ (lemniscatic elliptic curve).

Proof. In these cases, the operator $L(0)$ has a symmetry under the groups $\mathbb{Z}_{3}, \mathbb{Z}_{6}$, and $\mathbb{Z}_{4}$, respectively. This symmetry easily implies the triviality of monodromy at 0 .

Remark 2.8. Case $(i)$ is well known and due to Hermite. Case (ii) was done by Halphen [12, p. 571] in the case of equal gaps; his proof easily extends to the case of general gaps and general $n$. For multivariable generalizations of Proposition 2.7, see [8].

Note that the operators $L(0)-\lambda$, where $L(0)$ is as in Proposition $2.7(i)-($ iii $)$ are the only operators $L$ which are symmetric under $\mathbb{Z}_{n}$ (where $n$ is the order of $L$ ), acting by $z \mapsto e^{2 \pi i / n} z$. We will call such operators fully cyclically symmetric (or just cyclically symmetric if there is no ambiguity (e.g., when $n$ is a prime)). 
Conjecture 2.9. For any $n \geq 2$, there exists $N \in \mathbb{Z}_{+}$such that if $q_{j} \geq N$ for all $j=1, \ldots, n-1$, then the only algebraically integrable operators $L$ with gaps $q_{j}$ are fully cyclically symmetric.

\section{Remark 2.10.}

1. Conjecture 2.9, in particular, claims that algebraic integrability for large enough gaps takes place only for $n=2,3,4,6$.

2. As we have explained, for $n=2$ the Conjecture 2.9 holds with $N=0$.

3. Conjecture 2.9 is open even for $n=3$. It is supported by computational evidence and partial results described in the next section.

A similar conjecture can be made in the rational case. Namely, assume that $a_{i}(z) \in \mathbb{C}\left[z^{-1}\right]$. In this case, we have fully cyclically symmetric operators $L_{0}-\lambda$ for any $n \geq 2$, which are algebraically integrable.

Conjecture 2.11. Conjecture 2.9 holds in the rational case.

Remark 2.12. In the case $n=2$, the differential equation $L \psi=\lambda \psi$ is confluent hypergeometric, and Conjecture 2.11 is well known to be true (with $N=0$ ).

\subsection{The classification of elliptic finite gap potentials}

In this subsection we give a proof of the classification theorem of finite-gap potentials on elliptic curves with arbitrary number of poles $[10,4,11]$; this proof is based on differential Galois theory and follows [4, Section 4.1].

Theorem 2.13 ([11]). Let $u(z)$ be a rational function on an elliptic curve $E$, which is a finite-gap potential (i.e., the operator $L=\partial^{2}+u$ is algebraically integrable). Then there exist nonnegative integers $m_{1}, \ldots, m_{N}$ and points $z_{1}, \ldots, z_{N} \in E$ satisfying the equations

$$
\sum_{j \neq i} m_{j}\left(m_{j}+1\right) \wp^{(2 s-1)}\left(z_{i}-z_{j}\right)=0, \quad i=1, \ldots, N, \quad s=1, \ldots, m_{i},
$$

such that

$$
u(z)=-\sum_{i=1}^{N} m_{i}\left(m_{i}+1\right) \wp\left(z-z_{i}\right)+\text { const. }
$$

Moreover, any potential of this form is finite-gap.

For the proof of this theorem, we will need the following classical lemma from the theory of differential equations.

Lemma 2.14. Let $m \in \mathbb{Z}_{+}$, and

$$
u(z)=-m(m+1) z^{-2}+\sum_{j=1}^{\infty} c_{j} z^{j} \in \mathbb{C}((z)) .
$$

Then the equation

$$
\left(\partial^{2}+u\right) \psi=\lambda \psi
$$

admits two linearly independent solutions in $\mathbb{C}((z))$ for all $\lambda \in \mathbb{C}$ if and only if $c_{1}=c_{3}=\cdots=$ $c_{2 m-1}=0$. 
Proof. We need to show that the given condition is equivalent to the existence of a solution of the form $\sum_{n>0} a_{n} z^{n-m}$ with $a_{0}=1$. If all odd-numbered coefficients $c_{j}$ are zero (the $\mathbb{Z}_{2^{-}}$ symmetric case), then the required solution clearly exists. Otherwise, the obstruction to the existence of such solution is a certain homogeneous polynomial $P$ of $c_{j}$ and $\lambda$ of degree $2 m+1$ (where $\operatorname{deg}\left(c_{j}\right)=j+2, \operatorname{deg}(\lambda)=2$ ). Suppose that $s$ is the smallest integer such that $c_{2 s-1}$ is nonzero. Then it is easy to compute that the polynomial $P$ has degree $m-s$ with respect to $\lambda$, and its leading term is a multiple of $c_{2 s-1} \lambda^{m-s}$. Thus, this polynomial is nonzero, and the required solution does not exist. On the other hand, if $s \geq m+1$, then $P=0$, and the required solution exists.

Proof of Theorem 2.13. Theorem 2.1 implies that the poles of $u$ must be exactly second order (it is clear that if $u$ has a first order pole then (2.2) does not admit a basis of meromorphic solutions). Moreover, it follows from Corollary 2.4 that the singular part of $u$ at each pole $z_{i}$, $i=1, \ldots, N$, is $-m_{i}\left(m_{i}+1\right)\left(z-z_{i}\right)^{-2}$, where $m_{i}$ is a nonnegative integer. Thus, we find that in the algebraically integrable case

$$
u(z)=-\sum_{i=1}^{N} m_{i}\left(m_{i}+1\right) \wp\left(z-z_{i}\right)+\text { const. }
$$

Let us now show that the conditions for algebraic integrability in terms of $m_{i}, z_{i}$ are exactly as stated in the theorem. According to Theorem 2.5, the condition for algebraic integrability is that the monodromy of $(2.2)$ around each pole of $u$ is trivial. So the theorem follows from Lemma 2.14.

In particular, if all $m_{i}=1$, we obtain the following well known result:

Corollary 2.15 ([1]). The potential $u=-2 \sum \wp\left(z-z_{i}\right)$ is algebraically integrable if and only if $\left(z_{1}, \ldots, z_{N}\right)$ is a critical point of the elliptic Calogero-Moser potential

$$
U\left(z_{1}, \ldots, z_{N}\right)=\sum_{1 \leq j \neq i \leq N} \wp\left(z_{i}-z_{j}\right) .
$$

Remark 2.16. The same method can be used to rederive the classification from [10] of trigonometric and rational finite-gap potentials which are bounded at infinity, i.e., potentials on the nodal and the cuspidal curve of arithmetic genus $1 .^{2}$ It leads to the same answer, with $\wp(z)$ replaced by $\frac{1}{\sin ^{2} z}$ and $\frac{1}{z^{2}}$, respectively.

Note that (as explained in [10] and references therein), in the rational case we have the identity

$$
\sum_{i} m_{i}\left(m_{i}+1\right)=m(m+1)
$$

for some $m \in \mathbb{Z}_{+}$. This identity comes from the fact that a rational potential

$$
u(z)=-\sum_{i} \frac{m_{i}\left(m_{i}+1\right)}{\left(z-z_{i}\right)^{2}}
$$

can be degenerated into

$$
u_{0}(z)=-\frac{\sum_{i} m_{i}\left(m_{i}+1\right)}{z^{2}}
$$

\footnotetext{
${ }^{2}$ As explained in [11], boundedness at infinity is in fact automatic.
} 
by rescaling $z$ (so that algebraic integrability of $\partial^{2}+u$ implies algebraic integrability of $\partial^{2}+u_{0}$ ). We see that, surprisingly, identity (2.4) follows automatically from the existence of solutions of the equations

$$
\sum_{j \neq i} \frac{m_{j}\left(m_{j}+1\right)}{\left(z_{i}-z_{j}\right)^{2 s+1}}=0, \quad i=1, \ldots, N, \quad s=1, \ldots, m_{i} .
$$

\section{Third order operators with one pole}

\subsection{The general setup}

Let us now consider in detail the case $n=3$ with one pole at 0 . Up to an additive constant, the operator $L$ in this case looks like

$$
L=\partial^{3}+(a \wp(z)+c) \partial+\left(b \wp^{\prime}(z)+e \wp(z)\right),
$$

where $a, b, c, e \in \mathbb{C}$.

As explained above, a necessary condition for algebraic integrability is that the indices $m_{0}<$ $m_{1}<m_{2}$ at 0 are integers pairwise distinct modulo 3. So we have $m_{0}+m_{1}+m_{2}=3$, and

$$
a=m_{0} m_{1}+m_{0} m_{2}+m_{1} m_{2}-2, \quad b=\frac{1}{2} m_{1} m_{2} m_{3} .
$$

As above, it is convenient to introduce the "gaps" $q=q_{1}=m_{1}-m_{0}, r=q_{2}=m_{2}-m_{1}$ (then $\left.m_{0}=1-(2 q+r) / 3, m_{1}=1+(q-r) / 3, m_{2}=1+(2 r+q) / 3\right)$.

Our goal is to determine the algebraic integrability locus for each set $m_{0}, m_{1}, m_{2}$ in terms of the homogeneous coordinates $c, e, g_{2}, g_{3}$.

Recall that the $j$-invariant of $E$ is defined by the formula

$$
j(E)=\frac{1728 g_{2}^{3}}{g_{2}^{3}-27 g_{3}^{2}} .
$$

In particular, the equianharmonic (i.e., $\mathbb{Z}_{3}$-symmetric) elliptic curve $E$ has $g_{2}=0$ and $j=0$. Thus, by Theorem 2.7, the point $c=e=g_{2}=0$ belongs to the algebraic integrability locus for any $q, r$.

Note that the parameters $a, b$ of the operator $-L^{*}$, where $L^{*}$ is the adjoint operator to $L$, are given by the formulas $a^{\prime}=a, b^{\prime}=-b+a$ (and also $c^{\prime}=c, e^{\prime}=-e$ ). So the indices of $L^{*}$ are $2-m_{i}, i=0,1,2$, and hence the gaps $q$ and $r$ are interchanged under passing to the adjoint operator. On the other hand, it is clear that $L^{*}$ is algebraically integrable if and only if so is $L$. So it suffices to consider the case $q \geq r$.

Also note that the gaps cannot be divisible by 3 , and must be equal modulo 3 . So we can write $q=r+3 k$, where $k \geq 0$ is an integer.

We have a basis of solutions of equation (2.2) of the form

$$
\psi_{i}(z)=z^{m_{i}}(1+o(1)), \quad z \rightarrow 0 .
$$

Obviously, $\psi_{2}$ is single-valued near 0 ; algebraic integrability of $L$ is equivalent to the condition that $\psi_{0}(z), \psi_{1}(z)$ are single-valued near 0 , i.e., do not contain log factors. However, it turns out that even a weaker condition suffices. Namely, we have the following important proposition.

Proposition 3.1. If $\psi_{1}$ is single-valued near 0 for all $\lambda$, then so is $\psi_{0}$, and thus $L$ is algebraically integrable.

The proof of Proposition 3.1 is based on the following well known lemma from linear algebra. 
Lemma 3.2. If $A, B$ are two square matrices such that $A B-B A$ has rank at most 1 then $A, B$ are simultaneously upper triangular in some basis.

Proof. Without loss of generality, we can assume ker $A \neq 0$ (by replacing $A$ with $A-\lambda$ if needed) and that $A \neq 0$. It suffices to show that there exists a proper nonzero subspace invariant under $A, B$; then the statement will follow by induction in dimension.

Let $C=[A, B]$ and suppose $\operatorname{rank} C=1$ (since the case $\operatorname{rank} C=0$ is trivial). If $\operatorname{ker} A \subset \operatorname{ker} C$, then $\operatorname{ker} A$ is $B$-invariant: if $A v=0$ then $A B v=B A v+C v=0$. Thus ker $A$ is the required subspace. If $\operatorname{ker} A \nsubseteq \operatorname{ker} C$, then there exists a vector $v$ such that $A v=0$ but $C v \neq 0$. So $A B v=C v \neq 0$. Thus $\operatorname{Im} C \subset \operatorname{Im} A$. So $\operatorname{Im} A$ is $B$-invariant: $B A v=A B v+C v \in \operatorname{Im} A$. So $\operatorname{Im} A$ is the required subspace.

This proves the lemma.

Corollary 3.3. If $A, B$ are two invertible square matrices such that $A B A^{-1} B^{-1}-1$ has rank at most 1 then $A, B$ are simultaneously upper triangular in some basis.

Proof. Let $A B A^{-1} B^{-1}-1=T$, where $T$ has rank at most 1 . Then $A B-B A=T B A$, and $T B A$ has rank at most 1 . So the result follows from the lemma.

Proof of Proposition 3.1. Let $A$ and $B$ be the monodromy matrices of (2.2) (for some base point) along the global cycles of the elliptic curve. Then $A B A^{-1} B^{-1}=U$, where $U$ is unipotent. Since $\psi_{1}$ is single valued, $U-1$ has rank 1 . So it follows from Corollary 3.3 that the monodromy of (2.2) is upper triangular, so the result follows from Theorem 2.5.

Using Proposition 3.1 and CAS "Maple", we computed the algebraic integrability locus for small values of $r$ and any value of $q$. In each case, we applied $L-\lambda$ to a generic function

$$
z^{m_{1}}\left(1+f_{1} z+f_{2} z^{2}+\cdots+f_{r} z^{r}+O\left(z^{r+1}\right)\right)
$$

solved for $f_{1}, \ldots, f_{r-1}$, and look at the coefficient of $z^{m_{1}+r}$ in the result. This is a polynomial in $\lambda$, and by Proposition 3.1, the monodromy around 0 is trivial iff the coefficients of this polynomial vanish; this gives equations in $c, e, g_{2}, g_{3}$.

Remark 3.4. As explained above, homothety of the elliptic curve rescales $c, e, g_{2}, g_{3}$ and $\lambda$, giving a natural notion of degree:

$$
\operatorname{deg}(e)=1, \quad \operatorname{deg}(c)=2, \quad \operatorname{deg}\left(g_{2}\right)=4, \quad \operatorname{deg}\left(g_{3}\right)=6, \quad \operatorname{deg}(\lambda)=3,
$$

and the constraint polynomial is homogeneous of degree $r$.

The results of our computations are presented in the next subsection.

\subsection{Results of computer calculations}

\subsection{1 $r=1$}

The coefficient of $z^{m_{1}+1}$ in the image of $L-\lambda$ is $e$, and thus the operator is algebraically integrable iff $e=0$; this gives a 1-parameter family of integrable operators on each elliptic curve. The case $q=1$ is trivial $\left(L=\partial^{3}+c \partial\right)$, while the case $q=4$ was considered by Picard in 1881. 


\section{$3.2 .2 r=2$}

The coefficient of $z^{m_{1}+2}$, after solving for $f_{1}$, is independent of $\lambda$, and thus gives an equation relating $c$ and $e$ :

$$
c=-\frac{3 e^{2}}{(q+1)^{2}}
$$

Each curve thus has a 1-parameter family of integrable operators of this form.

In particular, setting $q=r, e=0$ we get Example 1 of [18]. This is the third order operator commuting with the Lame operator $\partial^{2}-2 \wp$.

\subsection{3 $r=4$}

Now the coefficient is linear in $\lambda$. The leading coefficient is a positive multiple of $e$, so $e=0$; then the constant coefficient relates $g_{2}$ and $c$ :

$$
c^{2}=\frac{(q+2)^{2}}{3} g_{2}
$$

Thus each elliptic curve admits a pair of integrable operators of this form, except that when $g_{2}=0$, the two operators coincide (with the corresponding cyclically symmetric operator).

In particular, setting $q=r, e=0$ we get Example 2 of [18].

\subsection{4 $r=5$}

Again the constraining polynomial is linear in $\lambda$. The leading coefficient implies

$$
c=-\frac{3\left(7 q^{2}+35 q+46\right)}{16(q+1)^{2}(q+4)^{2}} e^{2}
$$

at which point the constant coefficient factors, so that either $c=e=0$ or

$$
g_{2}=\frac{27\left(4 q^{2}+20 q+25\right)}{64(q+1)^{4}(q+4)^{4}} e^{4} .
$$

Each elliptic curve thus admits four integrable operators of this form (or two modulo the symmetry $z \rightarrow-z$ ) except the equianharmonic case $g_{2}=0$, where all these operators coincide with the cyclically symmetric operator.

In particular, setting $q=r, e=0$ we get Example 3 of [18].

\subsection{5 $r=7$}

The constraint polynomial has degree 2 , with leading coefficient

$$
\frac{5(q+2)(q+5)}{144(q+1)(q+3)(q+4)(q+6)} e,
$$

so that $e$ must be 0 . But this also eliminates the constant term, leaving only an equation relating $c$ and $g_{2}$ :

$$
c^{2}=\frac{25(q+2)^{2}(q+5)^{2}}{12(2 q+7)^{2}} g_{2} .
$$

So we get two operators on each elliptic curve which coincide in the $\mathbb{Z}_{3}$-symmetric case $g_{2}=0$ (with the cyclically symmetric operator).

In particular, setting $q=r, e=0$ we get Example 4 of [18]. 


\subsection{6 $r=8$}

The constraint polynomial again has degree 2, and the leading coefficient implies

$$
c=-\frac{3\left(191 q^{4}+3056 q^{3}+17598 q^{2}+42992 q+38384\right)}{686(q+1)^{2}(q+4)^{2}(q+7)^{2}} e^{2} .
$$

Unlike in the previous case, however, one still has two conditions remaining, of weighted degrees 5 and 8 respectively. The degree 5 condition naturally has a factor of $e$, but setting $e=0$ makes $c=0$ so that the remaining constraint is a multiple of $g_{2}^{2}$; the coefficient is negative, so this makes $g_{2}=0$. In the remaining case, the degree 5 condition gives a formula for $g_{2}$ :

$$
g_{2}=\frac{27 g_{2}^{\text {num }}}{g_{2}^{\text {den }}} e^{4}
$$

where

$$
\begin{aligned}
g_{2}^{\text {num }}:= & 155383 q^{8}+4972256 q^{7}+68978821 q^{6}+541706360 q^{5}+2632855228 q^{4} \\
& +8104425920 q^{3}+15416669104 q^{2}+16555419008 q+767835508
\end{aligned}
$$

and

$$
g_{2}^{\mathrm{den}}=470596(q+1)^{4}(q+4)^{4}(q+7)^{4}\left(19 q^{2}+152 q+277\right),
$$

and the degree 8 condition can then be solved for $g_{3} / e^{6}$. For each $q=8+3 k, k \geq 0$, one thus has a single $j$-invariant other than $j=0$ for which there exists an algebraically integrable operator:

$$
j=-6912 \frac{j^{\text {num }}}{j^{\text {den }}},
$$

where

$$
j^{\text {num }}=p_{8}(q)^{3}\left(19 q^{2}+152 q+277\right),
$$

with $^{3}$

$$
\begin{aligned}
p_{8}(q)= & 155383 q^{8}+4972256 q^{7}+68978821 q^{6}+541706360 q^{5}+2632855228 q^{4} \\
& +8104425920 q^{3}+15416669104 q^{2}+16555419008 q+7678355008
\end{aligned}
$$

and

$$
\begin{aligned}
j^{\text {den }}= & (q+7)(q+6)(q+2)(q+1)\left(67 q^{2}+533 q+898\right)\left(67 q^{2}+539 q+922\right) \\
& \times\left(37 q^{3}+399 q^{2}+1344 q+1468\right)\left(37 q^{3}+489 q^{2}+2064 q+2692\right) \\
& \times\left(367 q^{3}+5115 q^{2}+23376 q+34828\right)\left(367 q^{3}+3693 q^{2}+12000 q+12724\right) \\
& \times\left(829 q^{4}+14194 q^{3}+89097 q^{2}+242068 q+239236\right) \\
& \times\left(829 q^{4}+12334 q^{3}+66777 q^{2}+156028 q+133156\right) .
\end{aligned}
$$

Note that $j$ is finite for any $q$ since the factors of $j^{\text {den }}$ have positive coefficients.

In particular, for $e=0$ the only solution is $g_{2}=0$, which is shown for $q=r$ in Example 5 of $[18]$.

\footnotetext{
${ }^{3}$ We note that $p_{8}$ is the numerator of $g_{2} / e^{4}$.
} 


\subsection{7 $r=10$}

The constraint polynomial is cubic in $\lambda$, and the leading coefficient implies that $e=0$; setting $e=0$ makes the polynomial even in $\lambda$, so one has two remaining constraints, of degrees 4 and 10 respectively. The degree 4 constraint can be solved for $g_{2}$ :

$$
g_{2}=\frac{3\left(2069 q^{4}+41380 q^{3}+301017 q^{2}+941170 q+1071464\right)}{4400(q+2)^{2}(q+5)^{2}(q+8)^{2}} c^{2},
$$

at which point the degree 10 constraint is $c^{2}$ times an equation for $g_{3}$. Thus either $e=c=g_{2}=0$ or

$$
g_{3}=-\frac{g_{3}^{\text {num }}}{g_{3}^{\text {den }}}
$$

where

$$
\begin{aligned}
g_{3}^{\text {num }}:= & 96577 q^{6}+2897310 q^{5}+35259207 q^{4}+222299140 q^{3} \\
& +764656215 q^{2}+1360455150 q+978817201
\end{aligned}
$$

and

$$
g_{3}^{\text {den }}:=422400(q+2)^{3}(q+5)^{2}(q+8)^{3} .
$$

Thus other than $j=0$, the only possible $j$ invariant is

$$
j=-995328 \frac{j^{\text {num }}}{j^{\text {den }}}
$$

where

$$
j^{\text {num }}:=\left(2069 q^{4}+41380 q^{3}+301017 q^{2}+941170 q+1071464\right)^{3}
$$

and

$$
\begin{aligned}
j^{\text {den }}= & (5 q+19)(5 q+31)(13 q+47)(13 q+83)(17 q+73)(17 q+97)(19 q+59) \\
& \times(19 q+131)\left(11 q^{2}+110 q+239\right)\left(23 q^{2}+200 q+317\right)\left(23 q^{2}+260 q+617\right) .
\end{aligned}
$$

\subsection{8 $r=11$}

The constraint polynomial is cubic in $\lambda$, and other than $c=e=g_{2}=0$, there is no solution to the resulting four equations; one can solve the first three for $c, g_{2}, g_{3}$ in terms of $e$, and plug in to the fourth equation, obtaining $e^{11}$ times a rational function which is negative for $q>8$. Thus $c=e=g_{2}=0$ is the only solution.

\subsection{9 $r=13$}

The constraint polynomial is quartic in $\lambda$, but again the first equation is $e=0$, and eliminates half of the remaining equations. One thus has two additional equations which can be solved to give either $c=g_{2}=0$ or $g_{2} / c^{2}, g_{3} / c^{3}$ equal to specific rational functions of $q$. There is thus again a single surviving $j$ invariant, which is given by the formula

$$
j=-124416 \frac{j^{\text {num }}}{j^{\text {den }}}
$$


where

$$
\begin{aligned}
j^{\text {num }}= & \left(67 q^{2}+871 q+2014\right)^{2}\left(24727 q^{6}+964353 q^{5}+15225009 q^{4}+124224139 q^{3}\right. \\
& \left.+551142996 q^{2}+1258400208 q+1155995968\right)^{3}
\end{aligned}
$$

and

$$
\begin{aligned}
j^{\text {den }}= & (13 q+68)(13 q+101)\left(19 q^{2}+265 q+796\right)\left(19 q^{2}+229 q+562\right) \\
& \times\left(83 q^{2}+1094 q+2936\right)\left(83 q^{2}+1064 q+2741\right)\left(47 q^{3}+924 q^{2}+5481 q+9532\right) \\
& \times\left(47 q^{3}+909 q^{2}+5286 q+8824\right)\left(547 q^{3}+14649 q^{2}+127758 q+360056\right) \\
& \times\left(547 q^{3}+6684 q^{2}+24213 q+26876\right)\left(11 q^{2}+143 q+332\right) .
\end{aligned}
$$

\subsubsection{0 $r=14$}

If $e=0$, then $c=g_{2}=0$; otherwise, one can solve the first three equations for $c / e^{2}, g_{2} / e^{4}, g_{3} / e^{6}$, at which point the fourth equation is again a negative multiple of $e^{11}$, so there is no additional solution. Similar arguments apply to $r=17,20$.

\subsubsection{1 $r=16$}

The first equation makes $e=0$, so that one has three additional equations. If $c=0$, then the next equation makes $g_{2}=0$; otherwise, one can solve the first two equations for $g_{2} / c^{2}, g_{3} / c^{3}$, at which point the remaining equation is a negative (for $q>13$ ) multiple of $c^{8}$, so no other solution exists. Similar arguments apply to $r=19,22$.

Remark 3.5. Observe that the polynomials $j^{\text {den }}$ in the cases $r=8,10,13$ split into many irreducible factors over $\mathbb{Q}$, whose leading coefficients are either 1 or primes. Moreover, the constant coefficients of the factors for $r=10$ are also primes, while for $r=8,13$ they are primes times a small (at most third) power of 2 . The number-theoretic roots of this peculiar behavior are mysterious to us. We burden the reader with the unwieldy expressions of the $j$-invariants in the hope that someone would help us demystify it.

Remark 3.6. The operators with $q=r$ for $r=10$ and $r=13$ exist only for special values of $j$, so they are not present in [18], which deals with the case of generic $j$ only.

\subsection{The classification conjecture}

On the basis of this data we make the following conjecture.

Conjecture 3.7. For $q \geq r \geq 14$, there are no algebraically integrable operators $L$ apart from the one which is $\mathbb{Z}_{3}$-symmetric (i.e. $c=e=g_{2}=0$ ). Thus, all the algebraically integrable third order operators $L$ with one pole are the ones described in this subsection.

This is a more precise version of Conjecture 2.9 for $n=3$, claiming that in this case one may take $N=14$.

Here is a partial result in the direction of this conjecture.

\section{Proposition 3.8.}

(i) If $r=3 s+1$, where $s \geq 0$ is an integer, then for any algebraically integrable operator $L$ of the form (3.1), one has $e=0$. 
(ii) Assume that $r=3 s+1$, where $s \geq 1$ is an integer. If $L$ is an algebraically integrable operator of the form (3.1) then $g_{2}=0$ if and only if $c=0$ (in which case $L$ is cyclically symmetric).

Proof. (i) We have already checked the case $s=0$ directly, so we may assume that $s>0$.

Algebraic integrability of the operator (3.1) is equivalent to the existence of three linearly independent solutions of the differential equation

$$
\partial^{3} f+\left(t^{2} a \wp(t z)+t^{2} c\right) \partial f+\left(t^{3} b \wp^{\prime}(t z)+t^{3} e \wp(t z)\right) f=f
$$

in $\mathbb{C}((z))$ for generic $t$; this is just the image of the original eigenvalue equation under a homothety $z \mapsto t z$ of scale $t=\lambda^{-1 / 3}$. For $t=0$, this has three independent solutions, each of which must deform to a solution for general $t$ with the same asymptotics at $z=0$. Let $\psi_{1}(z)$ be the middle solution,

$$
\psi_{1}(z)=z^{m_{1}} \sum_{k \geq 0} \frac{(z / 3)^{3 k}}{(1-r / 3)_{k}(1+q / 3)_{k} k !} .
$$

Then we need to be able to deform this to a solution of the form $\psi_{1}(z)+t G_{1}(z)+O\left(t^{2}\right)$, and we claim that this implies $e=0$. Plugging

$$
G_{1}(z)=\sum_{k \geq 0} c(k) z^{m_{1}+3 k+1}
$$

into the equation gives

$$
\begin{aligned}
& \sum_{k \geq 0}[(3 k+1)(3 k+1-r)(3 k+q+1) c(k)-c(k-1)] z^{m_{1}+3 k-2} \\
& \quad=-e \sum_{k \geq 0} \frac{z^{m_{1}+3 k-2}}{3^{3 k}(1-r / 3)_{k}(1+q / 3)_{k} k !}(3 k+1)(3 k+1-r)(3 k+q+1) c(k)-c(k-1) \\
& \quad=-\frac{e}{3^{3 k}(1-r / 3)_{k}(1+q / 3)_{k} k !} .
\end{aligned}
$$

Setting $c^{\prime}(k)=c(k) 3^{3 k}(1-r / 3)_{k}(1+q / 3)_{k} k$ !, we obtain

$$
(3 k+1)(r-3 k-1)(q+3 k+1) c^{\prime}(k)-3 k(r-3 k)(3 k+q) c^{\prime}(k-1)=e .
$$

It follows that $c^{\prime}(k)$ is a positive multiple of $e$ for $0 \leq k<(r-1) / 3$. Since the equation for $k=(r-1) / 3$ reads $-(r-1)(q+r-1) c^{\prime}((r-4) / 3)=e$, this gives a contradiction unless $e=0$.

(ii) We have already computed the case $s=1$ directly, so we may assume $s>1$. Also, it follows from part $(i)$ that $e=0$. The perturbed solution, if it exists, will thus have the form

$$
\psi_{1}(z)+t^{2} G_{1}(z)+t^{4} G_{2}(z)+O\left(t^{5}\right) .
$$

(Note that the odd degree terms vanish by symmetry.) To the same order, the differential equation reads

$$
\partial^{3} f+\left(\frac{a}{z^{2}}+c t^{2}+\frac{a g_{2} z^{2}}{20} t^{4}\right) \partial f+\left(-\frac{2 b}{z^{3}}+\frac{b g_{2} z}{10} t^{4}\right) f=f+O\left(t^{5}\right) .
$$

Writing

$$
G_{1}(z)=z^{m_{1}+2} \sum_{k} c^{\prime}(k) \frac{(z / 3)^{3 k}}{(1-r / 3)_{k}(1+q / 3)_{k} k !},
$$




$$
G_{2}(z)=z^{m_{1}+1} \sum_{k} d^{\prime}(k) \frac{(z / 3)^{3 k}}{(1-r / 3)_{k}(1+q / 3)_{k} k !}
$$

and substituting in, we find from the $t^{2}$ term that

$$
\begin{aligned}
& (3 k+2)(r-3 k-2)(q+3 k+2) c^{\prime}(k)-3 k(r-3 k)(q+3 k) c^{\prime}(k-1) \\
& =((q-r) / 3+3 k+1) c
\end{aligned}
$$

and thus $c^{\prime}(k)$ is a positive multiple of $c$ for $0 \leq k \leq(r-4) / 3$. We also find that

$$
\begin{aligned}
& (3 k+1)(r-3 k-1)(q+3 k+1) d^{\prime}(k)-3 k(r-3 k)(q+3 k) d^{\prime}(k-1) \\
& \quad=-k(r-3 k)(q+3 k)(q-r+9 k) c^{\prime}(k-1) c+k(r-3 k)(q+3 k) C(k, q, r) g_{2}
\end{aligned}
$$

where $C(k, q, r)$ is positive when $4 \leq 3 k+1 \leq r \leq q$, since the appropriate linear change of variables gives a polynomial with positive coefficients. We find by induction that $d^{\prime}(k)$ is a nonnegative linear combination of $-c^{2}$ and $g_{2}$ for $0 \leq k \leq(r-4) / 3$, while the equation for $k=(r-1) / 3$ also tells us that $d^{\prime}((r-4) / 3)$ is a negative linear combination of $-c^{2}$ and $g_{2}$. Subtracting the expressions for $d^{\prime}((r-4) / 3)$ gives a positive linear combination of $-c^{2}$ and $g_{2}$ which vanishes. In particular, if one of $c$ and $g_{2}$ is 0 , so is the other.

\subsection{The nodal and cuspidal cases}

The results of the previous subsections also apply to the nodal case $j=\infty$ and the cuspidal case $g_{2}=g_{3}=0$.

Namely, in the nodal case, we get 1-parameter families of algebraically integrable operators for $r=1,2$, finite collections operators for $r=4,5,7$, and conjecturally no solutions for larger $r$ (this is confirmed for $r \leq 22$ ).

In the cuspidal case $g_{2}=0, g_{3}=0$, we always have an algebraically integrable operator with cyclic symmetry; apart from that, we get 1-parameter families of algebraically integrable operators for $r=1,2$, and conjecturally no other cases (if $r=3 s+1$, this is true by Proposition 3.8).

\section{Operators with several poles}

\subsection{Third order operators}

Consider now a third order algebraically integrable operator

$$
L=\partial^{3}+a(z) \partial+b(z)
$$

on an elliptic curve $E$ with several poles $z_{1}, \ldots, z_{N} \in E$.

It is easy to show that if the gaps of $L$ at a given point are $q=r=1$, then the operator must be holomorphic at this point, i.e. this case is trivial. So we consider the simplest nontrivial case, when the gaps at all the poles are $q=r=2$.

Lemma 4.1. Let $L=\partial^{3}+a(z) \partial+b(z)$ be a Fuchsian differential operator near $z=0$ with gaps $q=r=2$. Let

$$
a(z)=\sum_{k \geq 0} a_{k} z^{k-2}, \quad b(z)=\sum_{k \geq 0} b_{k} z^{k-3} .
$$

Then $L-\lambda$ has trivial monodromy around 0 for any $\lambda$ if and only if the Laurent coefficients $a_{1}, b_{2}$ are zero, and

$$
a_{2}=-\frac{b_{1}^{2}}{3}, \quad b_{4}=a_{4}+\frac{b_{1} a_{3}}{3} .
$$


Proof. We have three gaps 2, 2, 4, and three conditions associated to them, which are of degrees $2,2,4$. The conditions of degree 2 say that $b_{2}=0$ and $a_{2}=-b_{1}^{2} / 3$. The condition of degree 4 is linear in $\lambda$. The leading coefficient in $\lambda$ is of degree 1 , and is a nonzero multiple of $a_{1}$, so we get the condition $a_{1}=0$. The constant coefficient is of degree 4 and gives $b_{4}=a_{4}+\frac{b_{1} a_{3}}{3}$.

Clearly, the same result holds for a Fuchsian differential operator defined near any point $z=z_{0}$. Thus, any algebraically integrable operator (4.1) with gaps $q=r=2$ at all poles would necessarily have to be of the form

$$
\partial^{3}+\left(c-3 \sum_{i=1}^{N} \wp\left(z-z_{i}\right)\right) \partial-\frac{3}{2} \sum_{i=1}^{N} \wp^{\prime}\left(z-z_{i}\right)+\sum_{i=1}^{N} 3 p_{i} \wp\left(z-z_{i}\right),
$$

where $p_{i}$ and $c$ are complex numbers (up to adding a constant). Let us find the conditions on the parameters $z_{i}, p_{i}, c$ for this operator to be algebraically integrable.

Proposition 4.2. The conditions for algebraic integrability of $L$ are

$$
c+3 p_{i}^{2}=3 \sum_{j \neq i} \wp\left(z_{i}-z_{j}\right), \quad i=1, \ldots, N,
$$

and

$$
\sum_{j \neq i}\left(p_{i}+p_{j}\right) \wp^{\prime}\left(z_{i}-z_{j}\right)=0, \quad i=1, \ldots, N .
$$

Proof. The proof is by direct calculation using Lemma 4.1.

Corollary 4.3. Let

$$
F(z, p)=\sum_{i=1}^{N} p_{i}^{3}-\frac{3}{2} \sum_{i \neq j}\left(p_{i}+p_{j}\right) \wp\left(z_{i}-z_{j}\right) .
$$

Then $L$ is algebraically integrable if and only if $(z, p)$ is a critical point of the function $F(z, p)+$ $c \sum_{i=1}^{N} p_{i}$.

We note that $F$ is the cubic integral $H_{3}$ for the elliptic Calogero-Moser Hamiltonian

$$
H_{2}=\sum_{i=1}^{N} p_{i}^{2}-\sum_{j \neq i} \wp\left(z_{i}-z_{j}\right) .
$$

Thus, the algebraically integrable operators for a fixed value of $c$ are the critical points of $H_{3}+c H_{1}$, where $H_{1}=\sum p_{i}$.

So, Proposition 4.2 can be viewed as a third order analog of Corollary 2.15.

Remark 4.4. Corollary 4.3 is, essentially, a special case of the elliptic analog of Proposition 6 of $[1$, p. 124].

\subsection{Higher order operators}

We expect that in a similar way one can deal with higher order operators, obtaining families of algebraically integrable operators parametrized by critical points of higher Calogero-Moser Hamiltonians. Specifically, we expect that if we take the $n$-th order operator $L$ with indices $-1,1, \ldots, n-2, n$ and poles $z_{1}, \ldots, z_{N}$ on an elliptic curve, then the algebraically integrable operators will correspond to critical points of a degree $n$ elliptic Calogero-Moser Hamiltonian. Similarly to Corollary 4.3, this should be a consequence of the methods of [1] and [14].

This result obviously has trigonometric and rational counterparts.

We note, however, that whether the corresponding variety of critical points is nonempty, what is its dimension, etc., are, in general, difficult questions. 


\subsection{Operators with symmetries}

It is also interesting to consider operators with symmetries. For example, suppose $L$ is a second order operator $\partial^{2}+u(z)$ which is even with respect to $z$. Assume that it has poles at the fixed points of $z \rightarrow-z$ (i.e. $w_{0}=0, w_{1}=1 / 2, w_{2}=\tau / 2$, and $w_{3}=(1+\tau) / 2$ ) and at some other distinct points $\pm z_{1}, \ldots, \pm z_{N}$. Assume that the indices of $L$ at the fixed points $w_{i}$ are $-m_{i}, m_{i}+1$ for $i=0,1,2,3$, and the indices at $\pm z_{j}$ are $-1,2$. In this case, similarly to Proposition 2.15, it is easy to show that algebraically integrable operators correspond to critical points of the Inozemtsev potential

$$
U:=\sum_{i=0}^{3} \sum_{j=1}^{N}\left(m_{i}+\frac{1}{2}\right)^{2} \wp\left(z_{j}-w_{i}\right)+\sum_{1 \leq k \neq j \leq N}\left(\wp\left(z_{j}-z_{k}\right)+\wp\left(z_{j}+z_{k}\right)\right)
$$

(see [17, Theorem 0.2] for the case $N=1$ ).

In the same vein, one may consider operators $L$ of order $\ell=3,4,6$ which are invariant under

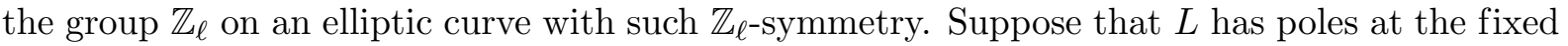
points $\eta_{j}$ of $\mathbb{Z}_{\ell}$, and also at some other points $z_{1}, \ldots, z_{N}$ (taken from distinct $\mathbb{Z}_{\ell}$-orbits) as well as their images under the $\mathbb{Z}_{\ell}$ action. Let us fix the indices at $\eta_{j}$ to be the same as the indices of the operator $L_{0 j}^{\ell / \ell_{j}}$, where $\ell_{j}$ is the order of the stabilizer of $\eta_{j}$, and $L_{0 j}$ is a rational homogeneous operator (2.1) of order $\ell_{j}$ with arbitrary integer indices. Also, let us fix the indices at the other poles to be $-1,1, \ldots, \ell-2, \ell$.

Conjecture 4.5. Algebraically integrable operators $L$ as above correspond to critical points of the lowest degree (i.e., degree $\ell$ ) Hamiltonian of the classical crystallographic elliptic CalogeroMoser system for the group $\mathbb{Z}_{\ell}$ (with appropriate parameters) defined in [8].

Remark 4.6. Conjecture 4.5 may be generalized to the case when the indices of $L_{0 j}$ are not assumed to be integers. Namely, in this case we conjecture that operators $L$ with trivial monodromy of $L \psi=\lambda \psi$ around non-fixed points of $\mathbb{Z}_{\ell}$ (i.e. those for which the monodromy gives rise to a representation of a generalized DAHA of rank 1 of type $E_{6}, E_{7}, E_{8}$ defined in [9]) correspond to critical points of the lowest degree Hamiltonian of the classical crystallographic elliptic Calogero-Moser system for the group $\mathbb{Z}_{\ell}$ with generic parameters.

Proposition 4.7. Conjecture 4.5 holds for $\ell=3$.

Proof. In the case $\ell=3$, the classical crystallographic elliptic Calogero-Moser Hamiltonian of [8] has the form

$$
H=\sum_{i=1}^{N} p_{i}^{3}+\sum_{i=1}^{N} \sum_{r=0}^{2}\left(A_{r} \wp\left(z_{i}-\eta_{r}\right) p_{i}+B_{r} \wp^{\prime}\left(z_{i}-\eta_{r}\right)\right)-3 C \sum_{i \neq j} \sum_{s=0}^{2} \wp\left(z_{i}-\varepsilon^{s} z_{j}\right) p_{i}
$$

where $\tau=\varepsilon:=e^{2 \pi \mathrm{i} / 3}, \wp(x):=\wp(x, \tau), \eta_{0}=0, \eta_{1}=\mathrm{i} \sqrt{3} / 3, \eta_{2}=-\mathrm{i} \sqrt{3} / 3$, and $A_{l}, B_{l}, C$ are parameters.

On the other hand, consider the $\mathbb{Z}_{3}$-symmetric operator

$$
\begin{aligned}
L= & \partial^{3}+\sum_{r=0}^{2}\left(\alpha_{r} \wp\left(z-\eta_{r}\right) \partial+\beta_{r} \wp^{\prime}\left(z-\eta_{r}\right)\right)-3 \sum_{i=1}^{N} \sum_{s=0}^{2} \wp\left(z-\varepsilon^{s} z_{i}\right) \partial \\
& +\sum_{i=1}^{N} \sum_{s=0}^{2}\left(-\frac{3}{2} \wp^{\prime}\left(z-\varepsilon^{s} z_{i}\right)+3 p_{i} \varepsilon^{-s} \wp\left(z-\varepsilon^{s} z_{i}\right)\right) .
\end{aligned}
$$


Using Lemma 4.1 and the identity

$$
\wp\left(\left(1-\varepsilon^{ \pm 1}\right) z\right)=-\frac{\varepsilon^{\mp 1}}{3}\left(\wp(z)+\wp\left(z-\eta_{1}\right)+\wp\left(z-\eta_{2}\right)\right),
$$

we obtain the following conditions for the operator $L$ to be algebraically integrable:

$$
\begin{gathered}
3 p_{i}^{2}=3 \sum_{j \neq i} \sum_{s=0}^{2} \wp\left(z_{i}-\varepsilon^{s} z_{j}\right)-\sum_{r=0}^{2}\left(\alpha_{r}-1\right) \wp\left(z_{i}-\eta_{r}\right), \\
\sum_{r=0}^{2}\left(\left(\alpha_{r}-1\right) \wp^{\prime}\left(z_{i}-\eta_{r}\right) p_{i}+\left(\frac{1}{2} \alpha_{r}-\beta_{r}\right) \wp^{\prime \prime}\left(z_{i}-\eta_{r}\right)\right) \\
=3 \sum_{j \neq i} \sum_{s=0}^{2} \wp^{\prime}\left(z_{i}-\varepsilon^{s} z_{j}\right)\left(p_{i}+\varepsilon^{-s} p_{j}\right),
\end{gathered}
$$

for $i=1, \ldots, N$. But these are exactly the conditions for a critical point of $H$, with $A_{r}=\alpha_{r}-1$, $B_{r}=\frac{1}{2} \alpha_{r}-\beta_{r}$, and $C=1$.

Remark 4.8. Similarly to the previous subsection, we expect that by considering operators $L$ of order $n \ell, n>1$, with $\mathbb{Z}_{\ell}$ symmetry, one can obtain families of algebraically integrable operators parametrized by critical points of a Hamiltonian of degree $n \ell$ (in momenta) for the crystallographic elliptic Calogero-Moser system of [8].

It would also be interesting to interpret the complete flow of this system (not only its critical points) along the lines of [14].

Remark 4.9. Here is a rational version of Conjecture 4.5, which allows arbitrary $\ell$. Namely, let

$$
L=\partial^{\ell}+a_{2}(z) \partial^{\ell-2}+\cdots+a_{\ell}(z)
$$

be a differential operator with rational coefficients, which is invariant under $\mathbb{Z}_{\ell}$, such that $a_{i}(z)$ vanish at infinity. Let the nonzero poles of $L$ be $z_{1}, \ldots, z_{N}$ (taken from different $\mathbb{Z}_{\ell \text {-orbits) as }}$ well as their images under the symmetry. Suppose that $L$ has arbitrary integer indices at 0 , and indices $-1,1, \ldots, \ell-2, \ell$ at $z_{1}, \ldots, z_{N}$.

Conjecture 4.10. Algebraically integrable operators $L$ with such properties correspond to critical points of the rational Calogero-Moser Hamiltonian (of degree $\ell$ ) for the complex reflection group $S_{N} \ltimes \mathbb{Z}_{\ell}^{N}$ (see e.g. [8]).

It follows from the above that this conjecture holds for $\ell \leq 3$.

Moreover, we expect that a similar conjecture holds for operators of order $n \ell$ with $\mathbb{Z}_{\ell^{-}}$ symmetry. Namely, in this case we should require that the indices at 0 are those of $L_{0}^{n}$, where $L_{0}$ is a rational homogeneous operator of order $\ell$ with integer indices, and we conjecture that algebraically integrable operators are parametrized by critical points of the higher order rational Calogero-Moser Hamiltonian (of order $n \ell$ ).

\section{Acknowledgements}

The authors are grateful to I. Krichever, E. Previato, and A. Veselov for useful discussions. The work of P.E. was partially supported by the the NSF grants DMS-0504847 and DMS-0854764. The work of E.R. was partially supported by the NSF grant DMS-1001645. 


\section{References}

[1] Airault H., McKean H.P., Moser J., Rational and elliptic solutions of the Korteweg-de Vries equation and a related many-body problem, Comm. Pure Appl. Math. 30 (1977), 95-148.

[2] Burchnall J.L., Chaundy T.W., Commutative ordinary differential operators, Proc. London Math. Soc. Ser. 221 (1923), 420-440.

[3] Braverman A., Etingof P., Gaitsgory D., Quantum integrable systems and differential Galois theory, Transform. Groups 2 (1997), 31-56, alg-geom/9607012.

[4] Chalykh O., Etingof P., Oblomkov A., Generalized Lamé operators, Comm. Math. Phys. 239 (2003), 115153, math.QA/0212029.

[5] Chalykh O., Algebro-geometric Schrödinger operators in many dimensions, Philos. Trans. R. Soc. Lond. Ser. A Math. Phys. Eng. Sci. 366 (2008), no. 1867, 947-971.

[6] Dubrovin B.A., Matveev V.B., Novikov S.P., Nonlinear equations of Korteweg-de Vries type, finite-band linear operators and Abelian varieties, Russ. Math. Surv. 31 (1976), no. 1, 59-146.

[7] Drinfel'd V.G., Krichever I.M., Manin Yu.I., Novikov S.P., Methods of algebraic geometry in contemporary mathematical physics, in Mathematical Physics Reviews, Soviet Sci. Rev. Sect. C: Math. Phys. Rev., Vol. 1, Harwood Academic, Chur, 1980, 1-54.

[8] Etingof P., Felder G., Ma X., Veselov A., On elliptic Calogero-Moser systems for complex crystallographic reflection groups, arXiv:1003.4689.

[9] Etingof P., Oblomkov A., Rains E., Generalized double affine Hecke algebras of rank 1 and quantized del Pezzo surfaces, Adv. Math. 212 (2007), 749-796, math.QA/0406480.

[10] Gesztesy F., Weikard R., Picard potentials and Hill's equation on a torus, Acta Math. 176 (1996), 73-107.

[11] Gesztesy F., Unterkofler K., Weikard R., An explicit characterization of Calogero-Moser systems, Trans. Amer. Math. Soc. 358 (2006), 603-656.

[12] Halphen G.H., Traité des fonctions elliptiques et de leurs applications, Vol. 2, Paris, 1888.

[13] Krichever I.M., Integration of nonlinear equations by methods of algebraic geometry, Funct. Anal. Appl. 11 (1977), no. 1, 12-26.

[14] Krichever I.M., Elliptic solutions of the Kadomcev-Petviashvili equations, and integrable systems of particles, Funct. Anal. Appl. 14 (1980), no. 4, 282-290.

[15] Previato E., Seventy years of spectral curves: 1923-1993, in Integrable Systems and Quantum Groups (Montecatini Terme, 1993), Lecture Notes in Math., Vol. 1620, Springer, Berlin, 1996, 419-481.

[16] Segal G., Wilson G., Loop groups and equations of KdV type, Inst. Hautes Études Sci. Publ. Math. (1985), no. $61,5-65$.

[17] Treibich A., Hyperelliptic tangential covers and finite-gap potentials, Russ. Math. Surv. 56 (2001), no. 6, 1107-1151.

[18] Unterkofler K., On the solutions of Halphen's equation, Differential Integral Equations 14 (2001), 1025-1050.

[19] Weikard R., On commuting differential operators, Electron. J. Differential Equations 2000 (2000), no. 19, 11 pages. 